\title{
PROBABILIDADE DE PRECIPITAÇÃO PARA A MICRORREGIÃO DE TANGARÁ DA SERRA, ESTADO DO MATO GROSSO ${ }^{1}$
}

\author{
Juliano Araújo Martins ${ }^{2}$, Rivanildo Dallacort ${ }^{3}$, Miriam Hiroko Inoue ${ }^{3}$, \\ Adalberto Santi ${ }^{3}$, Evandro Marcos Kolling ${ }^{4}$, Admar Júnior Coletti ${ }^{3}$
}

\section{ABSTRACT \\ PROBABILITY OF PRECIPITATION \\ FOR THE MICROREGION OF TANGARÁ DA SERRA, MATO GROSSO STATE, BRAZIL}

Due to the irregular occurrence of rainfall in Brazilian Savannas, associated with a vast range of models that aim to evaluate it, this study was carried out to estimate the monthly amount of rainfall, at different probability levels, in Tangará da Serra, Mato Grosso State, Brazil, by using the Gamma function. Pluviometric data from 1970 to 2007 (38 years), provided by the Agência Nacional de Águas (ANA), collected in a pluviometric station located at $14^{\circ} 37^{\prime} 55^{\prime} \mathrm{S}, 57^{\circ} 28^{\prime} 05^{\prime} \mathrm{W}$, and $488 \mathrm{~m}$ of altitude, were used. The adjustment analysis between estimated and observed data was carried out in accordance with the Kolmogorov-Smirnov adherence test, at $5 \%$ of significance, resulting in no significant differences for monthly periods, including those with several values equal or close to zero, allowing the Gamma distribution use. The highest expected monthly precipitation levels took place from November to March (rainy season), while from June to August were noticed the lowest ones (dry period). The other months can be considered as intermediaries between the dry and rainy seasons.

KEY-WORDS: Gamma distribution; expected precipitation; agricultural planning.

\section{INTRODUÇÃO}

De todos os segmentos da economia, a agricultura é o que apresenta maior dependência das variáveis climáticas, o que ocasiona oscilações de produção, ao longo dos anos. Dentre todas as variáveis meteorológicas, a precipitação se destaca como uma das principais responsáveis por tais variações. O conhecimento prévio de como a precipitação plu-

\section{RESUMO}

Devido à irregularidade na ocorrência de chuvas na região dos Cerrados, aliada aos inúmeros modelos que visam a avaliar tal parâmetro, objetivou-se, neste trabalho, estimar a precipitação pluviométrica mensal, em diferentes níveis de probabilidade, na região de Tangará da Serra (MT), utilizandose a Função Gama de Probabilidade. Foram utilizados dados pluviométricos de 1970 a 2007 (38 anos), disponibilizados pela Agência Nacional de Águas (ANA), coletados nas coordenadas $14^{\circ} 37^{\prime} 55^{\prime \prime}$ de latitude Sul, 57 $28^{\circ} 05^{\prime}$ de longitude Oeste e $488 \mathrm{~m}$ de altitude. Para verificação do ajuste entre os valores estimados e observados, utilizou-se o teste de aderência de Kolmogorov-Smirnov, ao nível de 5\% de significância, o qual não demonstrou discrepância significativa para períodos mensais, inclusive nos meses em que houve vários valores próximos ou iguais a zero, possibilitando, assim, a utilização da distribuição Gama. As maiores lâminas de precipitação esperadas ocorreram de novembro a março (estação chuvosa), enquanto, entre junho e agosto, ocorreram as menores lâminas de precipitação (estação seca). Os demais meses podem ser caracterizados como intermediários entre a estação seca e a chuvosa.

PALAVRAS-CHAVE: Distribuição Gama; precipitação esperada; planejamento agrícola.

viométrica se comportará é fundamental, visto que o sucesso das atividades agrícolas está diretamente ligado aos seus níveis de ocorrência (Smith 2000).

$\mathrm{Na}$ região dos Cerrados, as precipitações concentram-se no período primavera-verão, época em que se intensificam as atividades agrícolas. No entanto, nesta região, as chuvas apresentam grande irregularidade de ocorrência, seja espacial ou temporal, o que pode influenciar, significativamente, no

1. Trabalho recebido em jun./2009 e aceito para publicação em ago./2010 (nº registro: PAT 6365/ DOI: 10.5216/pat.v40i3.6365).

2. Escola Superior de Agricultura Luiz de Queiroz (ESALQ), Departamento de Engenharia e Biossistemas,

Piracicaba, SP, Brasil.E-mail: julianoaraujo@usp.br.

3. Universidade do Estado do Mato Grosso (Unemat), Campus Universitário de Tangará da Serra,

Departamento de Agronomia, Tangará da Serra, MT, Brasil.E-mails: rivanildo@unemat.br, miriamhinoue@hotmail.com, adalberto@unemat.br,admar_coletti@hotmail.com.

4. Universidade Tecnológica Federal do Paraná (IFET), Campus de Toledo, Coordenação de Engenharia Civil, Toledo, PR, Brasil. E-mail: kolling@utpr.edu.br. 
planejamento agrícola e na produtividade das culturas (Sousa 1998).

De acordo com Morais et al. (2001), no Brasil, os projetos de irrigação que não consideram a contribuição da precipitação no suprimento de água às culturas não se justificam economicamente, com exceção da região semiárida, uma vez que, nas demais regiões, a irrigação deve ter caráter complementar. Em alguns casos, utiliza-se o valor da precipitação média mensal no dimensionamento de sistemas de irrigação, o que pode resultar em subdimensionamento dos sistemas, podendo levar ao fracasso do agricultor (Frizzone 1979).

A escolha dos níveis de probabilidade de precipitação varia, dependendo da necessidade da cultura, do estádio de desenvolvimento e seu valor econômico, da disponibilidade de recursos hídricos e, sobretudo, das condições meteorológicas (Fietz et al. 1998). O nível de probabilidade geralmente recomendado para dimensionamento de sistemas de irrigação é 75\% (Bernardo 2008). Segundo Sampaio et al. (2007), o estudo probabilístico da distribuição pluviométrica desempenha papel relevante no planejamento racional da produção agrícola, auxiliando na predição da lâmina mínima a precipitar-se, com certa margem de segurança, e contribuindo para o melhor planejamento de irrigações suplementares, por meio do uso eficiente das águas, nas áreas cultivadas.

Com relação à agricultura de sequeiro, Stewart (1980) destaca que o desenvolvimento e rendimento das culturas dependem da época em que o período chuvoso tem início efetivo, bem como sua duração e regularidade. Machado et al. (1996), analisando a importância do comportamento da estação chuvosa, como fator limitante de produção, constataram relação entre duração do período chuvoso e potencial de produção agrícola, para a região norte de Minas Gerais.

Considerando-se a importância técnico-científica do assunto, aliada à ausência de dados referentes à probabilidade de precipitação na região, o objetivo deste trabalho foi analisar o ajuste da distribuição Gama, a fim de estimar a precipitação pluviométrica mensal esperada, em diferentes níveis de probabilidade, na região de Tangará da Serra (MT). Espera-se que estas informações possam auxiliar no dimensionamento de projetos de irrigação, construções de barragens, melhor planejamento de sistemas de manejos culturais, bem como no uso eficiente dos recursos hídricos.

\section{MATERIAL E MÉTODOS}

Foram utilizados dados de precipitação diária, disponibilizados pela Agência Nacional de Águas (ANA), coletados em estação pluviométrica localizada no município de Tangará da Serra, MT (latitude $14^{\circ} 37^{\prime} 55^{\prime} \mathrm{S}$, longitude $57^{\circ} 28^{\prime} 05^{\prime} \mathrm{W}$ e altitude de $488 \mathrm{~m}$ ). Em virtude da disponibilidade de dados, utilizou-se a série histórica de 1970 a 2007 (38 anos).

Os dados foram tabulados com posterior verificação de consistência, com auxílio do software computacional CLIMA, desenvolvido pelo Instituto Agronômico do Paraná (Faria et al. 2003). A determinação de diferentes níveis de probabilidade de precipitação foi realizada utilizando-se o modelo probabilístico de distribuição Gama incompleta (Thom 1958). Sua função densidade de probabilidade é dada pela equação

$$
G(y)=\frac{1}{\beta^{y} \times \Gamma(\alpha)} \times x^{\alpha-1} e^{-x / \beta},
$$

em que $\alpha=$ parâmetro de forma (adimensional); $\beta=$ parâmetro de escala (mm); $e=$ base do logaritmo neperiano; $\mathrm{x}=$ total de precipitação $(\mathrm{mm})$; e $\Gamma(\alpha)=$ símbolo da função Gama, definida conforme a equação

$$
\Gamma(\alpha)=\sqrt{2 \pi \alpha} \alpha^{\alpha} e^{\alpha}\left(1+\frac{1}{12 \alpha}+\frac{1}{288 \alpha^{2}}-\frac{139}{51840 \alpha^{3}}\right) .
$$

Os parâmetros que propiciam a distribuição Gama acumulada, para uma dada variável aleatória ( $\alpha$ e $\beta$ ), foram estimados pelo método dos momentos, conforme Thom (1958):

$$
\alpha=\frac{\bar{X}^{2}}{S^{2}} \quad \text { e } \quad \beta=\frac{S^{2}}{\bar{X}} .
$$

Para verificar o ajuste entre os dados estimados e observados, utilizou-se o teste de aderência de Kolmogorov-Smirnov, a 5\% de significância (Assis et al. 1996).

Posteriormente, as precipitações diárias de cada ano foram acumuladas em períodos mensais e, então, foram determinadas as médias, desvio-padrão e precipitações pluviométricas prováveis, aos níveis de $15 \%, 25 \%, 40 \%, 50 \%, 60 \%, 75 \%$ e $90 \%$ de probabilidade, para subsequente análise e discussão dos dados. 


\section{RESULTADOS E DISCUSSÃO}

Em análise prévia, observou-se que a região apresenta, no período em estudo, precipitação média anual de $1.830,8 \mathrm{~mm}$, sendo 1990 o ano mais chuvoso, com $2.861 \mathrm{~mm}$, enquanto 2004 foi o ano em que registrou-se a menor média de precipitação (1.404 mm). O desvio-padrão, para o referido período, foi de $312,8 \mathrm{~mm}$. A região apresenta estação seca e chuvosa bem definidas, uma vez que $32,11 \%$ das precipitações ocorrem na primavera; $47,14 \%$ no verão; $16,35 \%$ no outono; e $4,4 \%$ no inverno.

$\mathrm{Na}$ análise de probabilidade (Tabela 1), são apresentados os parâmetros da função Gama $(\alpha$, $\beta)$, resultados para o teste de aderência $(\mathrm{K}-\mathrm{S})$, probabilidade de não ocorrência de precipitação (Q) e médias e desvio-padrão mensais de precipitação, para o município de Tangará da Serra (MT). Observa-se que as variações máximas dos dados analisados pelo teste de Kolmogorov-Smirnov não ultrapassaram o valor crítico, a 5\% de significância (Tabela 1), indicando que a distribuição Gama se ajustou adequadamente aos dados, em todos os meses analisados. Resultados semelhantes foram encontrados por Morais et al. (2001), para o município de Lavras (MG); Murta et al. (2005), nos municípios de Itapetininga e Vitória da Conquista (BA); e Silva et al. (2007), para a região de Santa Maria (RS).
Observa-se que a variação interanual de precipitação é menos acentuada nos meses em que os valores de $\alpha$ são maiores, podendo este parâmetro ser utilizado na determinação de períodos regulares de precipitação. Por sua vez, os valores de $\beta$ não ultrapassaram, em nenhum dos meses, o valor crítico de 100. Segundo Thom (1958), tal condição possibilita a utilização da função Gama para determinação da precipitação provável, por meio da série de dados analisada. Conforme a Tabela 1, foram observados cinco meses durante o ano, com probabilidades da não ocorrência de precipitação $(\mathrm{Q})$, correspondendo aos meses de maio $(5,3 \%)$, junho $(31,6 \%)$, julho $(47,4 \%)$, agosto $(21,1 \%)$ e setembro $(2,6 \%)$.

$\mathrm{O}$ ajuste entre os dados estimados e observados é apresentado na Figura 1 (janeiro a dezembro). Os dados observados se ajustaram adequadamente ao modelo estimado, para todos os meses, mesmo naqueles que apresentaram valores de precipitação próximos a zero.

A precipitação mensal, em diferentes níveis probabilísticos (Tabela 2), indica que, para os níveis de $25 \%, 50 \%$ e $75 \%$ de probabilidade, são esperados valores acima de $250 \mathrm{~mm}, 200 \mathrm{~mm}$ e $150 \mathrm{~mm}$, respectivamente, para os meses de janeiro, fevereiro, março, novembro e dezembro. No entanto, para outubro e abril, esperam-se valores próximos a $200 \mathrm{~mm}$, $100 \mathrm{~mm}$ e $80 \mathrm{~mm}$, para os níveis de $25 \%, 50 \%$ e $75 \%$ de probabilidade, respectivamente.

Tabela 1. Parâmetros da função Gama $(\alpha, \beta)$, resultados para o teste de aderência (K-S), probabilidade de não ocorrência de precipitação (Q), médias e desvio-padrão mensais de precipitação (Tangará da Serra, MT, 1970 a 2007).

\begin{tabular}{lcccccrc}
\hline \multirow{2}{*}{ Mês } & $\alpha$ & \multirow{2}{*}{$\beta$} & \multicolumn{2}{c}{ K-S* } & \multirow{2}{*}{$\overline{\mathrm{X}}$} & $\mathrm{S}$ \\
& & & D.M. calc. & D.M.S & & & \\
\hline Janeiro & 7,39 & 42,43 & 0,082 & 0,215 & 0,000 & 313,46 & 115,32 \\
Fevereiro & 5,99 & 43,96 & 0,048 & 0,215 & 0,000 & 263,22 & 107,57 \\
Março & 5,70 & 46,08 & 0,063 & 0,215 & 0,000 & 262,48 & 109,98 \\
Abril & 3,44 & 47,53 & 0,073 & 0,215 & 0,000 & 163,52 & 88,16 \\
Maio & 1,03 & 59,36 & 0,080 & 0,215 & 0,053 & 60,98 & 60,16 \\
Junho & 0,49 & 21,23 & 0,047 & 0,215 & 0,316 & 10,33 & 14,81 \\
Julho & 0,43 & 26,17 & 0,082 & 0,215 & 0,474 & 11,27 & 17,18 \\
Agosto & 0,84 & 26,20 & 0,059 & 0,215 & 0,211 & 22,08 & 24,05 \\
Setembro & 1,56 & 47,37 & 0,046 & 0,215 & 0,026 & 73,74 & 59,10 \\
Outubro & 2,83 & 55,54 & 0,027 & 0,215 & 0,000 & 157,07 & 93,40 \\
Novembro & 4,88 & 44,57 & 0,046 & 0,215 & 0,000 & 217,62 & 98,48 \\
Dezembro & 17,39 & 15,82 & 0,071 & 0,215 & 0,000 & 275,10 & 65,97 \\
\hline
\end{tabular}

* K-S = Teste de Kolmogorov-Smirnov, a 5\% de significância. 

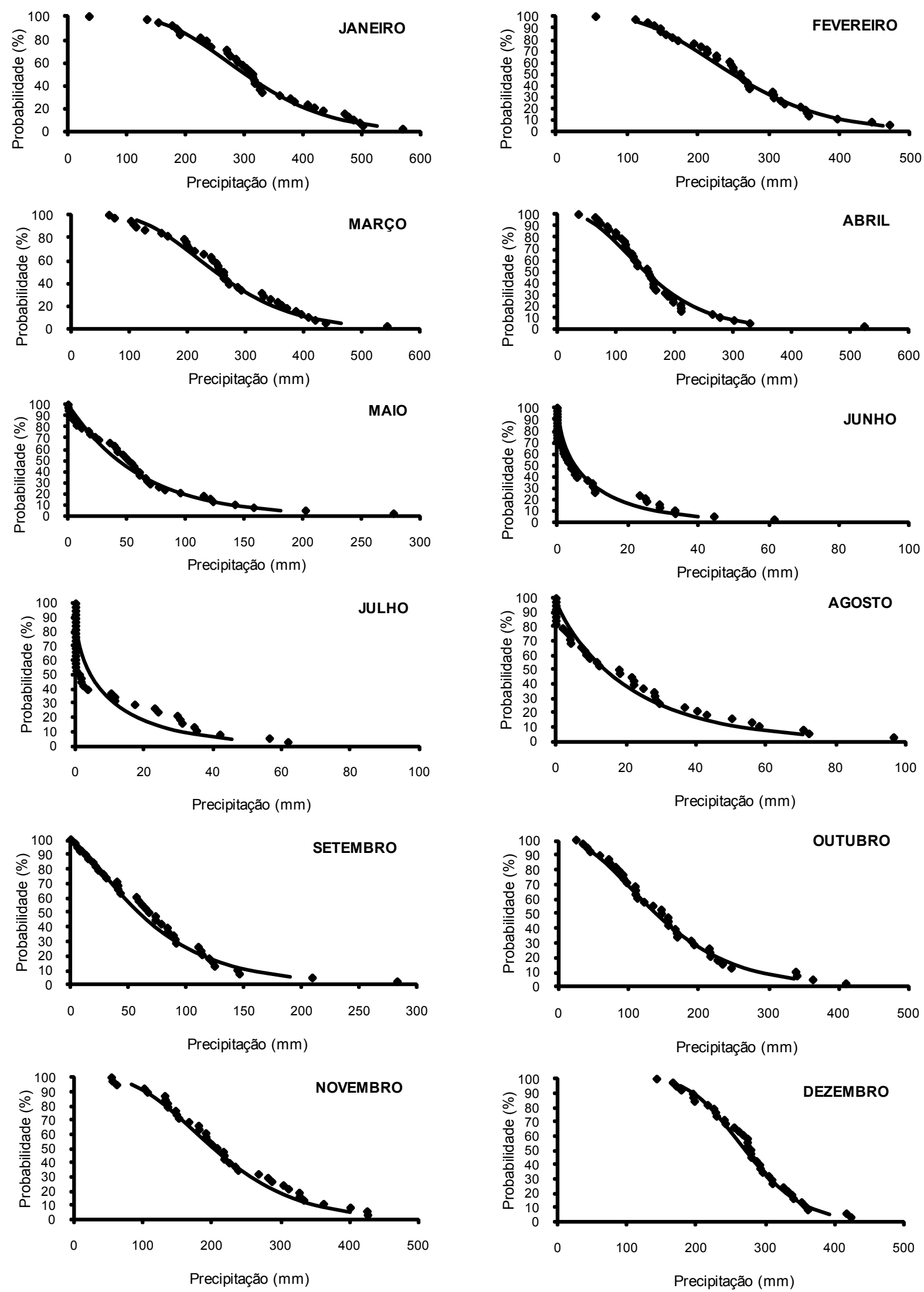

Figura 1. Ajuste dos valores reais de precipitação ao modelo de probabilidade, nos meses de janeiro a dezembro (Tangará da Serra, MT, 1970 a 2007). 
Tabela 2. Precipitação mensal esperada, em diferentes níveis de probabilidade (Tangará da Serra, MT, 1970 a 2007).

\begin{tabular}{lrrrrrrr}
\hline \multirow{2}{*}{ Mês } & \multicolumn{7}{c}{ Precipitação provável $(\mathrm{mm})$} \\
\cline { 2 - 7 } & \multicolumn{1}{c}{$10 \%$} & \multicolumn{1}{c}{$40 \%$} & \multicolumn{1}{c}{$50 \%$} & \multicolumn{1}{c}{$60 \%$} & \multicolumn{1}{c}{$75 \%$} & \multicolumn{1}{c}{$90 \%$} \\
\hline Janeiro & 467,33 & 381,70 & 328,79 & 299,43 & 271,89 & 229,98 & 177,70 \\
Fevereiro & 407,03 & 325,68 & 276,02 & 248,71 & 223,28 & 185,01 & 138,15 \\
Março & 409,58 & 326,04 & 275,19 & 247,29 & 221,36 & 182,45 & 135,04 \\
Abril & 281,74 & 211,40 & 170,06 & 147,98 & 127,92 & 98,83 & 65,51 \\
Maio & 139,48 & 84,52 & 56,21 & 42,71 & 31,65 & 18,02 & 6,75 \\
Junho & 28,10 & 13,60 & 7,20 & 4,58 & 2,74 & 0,99 & 0,15 \\
Julho & 31,41 & 14,49 & 7,28 & 4,44 & 2,52 & 0,81 & 0,09 \\
Agosto & 53,02 & 30,57 & 19,38 & 14,20 & 10,08 & 5,25 & 1,65 \\
Setembro & 152,28 & 100,79 & 72,75 & 58,69 & 46,62 & 30,56 & 15,03 \\
Outubro & 282,27 & 206,19 & 162,19 & 138,99 & 118,15 & 88,41 & 55,41 \\
Novembro & 349,49 & 273,60 & 227,86 & 202,96 & 179,95 & 145,74 & 104,75 \\
Dezembro & 362,30 & 316,27 & 286,71 & 269,85 & 253,66 & 228,21 & 194,67 \\
\hline
\end{tabular}

Nos meses de setembro e maio, a precipitação esperada, em níveis de $25 \%, 50 \%$ e $75 \%$, está em torno de $85 \mathrm{~mm}, 45 \mathrm{~mm}$ e $20 \mathrm{~mm}$, respectivamente. Já em junho, julho e agosto, a precipitação esperada, nestes mesmos níveis probabilísticos, são inferiores a valores de $30 \mathrm{~mm}$, $15 \mathrm{~mm}$ e $5 \mathrm{~mm}$, respectivamente (Tabela 2).

O mês de janeiro apresenta a maior precipitação provável, para os níveis de $25 \%, 50 \%$ e $75 \%$, com valores de $381,70 \mathrm{~mm}$; 299,43 $\mathrm{mm}$; e $229,98 \mathrm{~mm}$, respectivamente. Porém, para o nível de $90 \%$ de probabilidade, é o mês de dezembro que apresenta a maior precipitação esperada $(194,67 \mathrm{~mm})$. Este resultado sugere que, no mês de dezembro, existe maior regularidade de precipitação, pois a amplitude de variação dos valores máximos e mínimos de precipitação provável é menos acentuada que nos demais meses.

No período analisado, observa-se a existência de uma estação chuvosa na região, que se estende de outubro a abril, onde se concentram mais de $90 \%$ das precipitações. Contudo, mesmo apresentando altos índices pluviométricos, a utilização de sistemas de irrigação teria efeito benéfico, uma vez que existe variação interanual das precipitações, assim como má distribuição mensal das mesmas, ocasionando um fenômeno denominado veranico (Kumar \& Rao 2007). Segundo Bernardo (2008), as médias mensais de precipitações ocorridas, juntamente com o nível de probabilidade, são de grande importância na tomada de decisão, pelos agricultores, no manejo de irrigação, em meses de veranico.

Os meses de maio e setembro podem ser considerados de transição, concentrando $7,3 \%$ da precipitação média anual. No entanto, em alguns meses do ano, podem ocorrer valores de precipitação iguais ou próximos a $0 \mathrm{~mm}$. A estação seca se estende de junho a agosto, ocorrendo, em média, 2,7\% da precipitação anual. Estes valores são insuficientes para o desenvolvimento de qualquer cultura, sem a utilização de irrigação complementar, em especial as anuais, por possuírem sistema radicular geralmente superficial e não tolerarem estresse, devido aos ciclos curtos (Jadoski et al. 2007).

\section{CONCLUSÕES}

1. Utilizando-se o teste de Kolmogorov-Smirnov, a distribuição Gama ajustou-se, adequadamente, à série de dados em estudo, para períodos mensais.

2. A região apresenta estação seca e chuvosa bem definida, uma vez que $47,14 \%$ das precipitações ocorrem no verão; $16,35 \%$ no outono; $4,4 \%$ no inverno; e $32,11 \%$ na primavera.

3. Ao nível de $75 \%$ de probabilidade, os meses que apresentam maior probabilidade de precipitação são novembro a março, período em que a lâmina mensal esperada é superior a $150 \mathrm{~mm}$. No entanto, no mês mais chuvoso (janeiro), ao mesmo nível probabilístico, é esperada precipitação superior a 220 mm. Já nos meses de junho a agosto, a precipitação esperada, a 75\% de probabilidade, não ultrapassa 5,25 $\mathrm{mm}$ mensais.

\section{REFERÊNCIAS}

ASSIS, F. N.; ARRUDA, H. V.; PEREIRA, A. R. Aplicações de estatística à climatologia. Pelotas: Universitária, 1996. 
BERNARDO, S.; SOARES, A. A.; MANTOVANI, E. C. Manual de irrigação. 8. ed. Viçosa: UFV, 2008.

FARIA, R. T. et al. CLIMA: programa computacional para organização e análise de dados meteorológicos. Engenharia Agrícola, Jaboticabal, v. 23, n. 2, p. 372-387, 2003.

FIETZ, C. R. et al. Precipitação esperada, em diferentes níveis de probabilidade, na região de Dourados, MS. Ciência Rural, Santa Maria, v. 28, n. 1, p. 29-34, 1998.

FRIZZONE, J. A. Análise de cinco modelos para o cálculo da distribuição de frequência de precipitações na região de Viçosa, MG. 1979. 100 f. Dissertação (Mestrado em Irrigação e Drenagem)-Universidade Federal de Viçosa, Viçosa, 1979.

JADOSKI, S. O. et al. Efeito da lâmina de irrigação na senescência foliar do feijoeiro. Irriga, Botucatu, v. 12, n. 4 , p. 545-556, 2007.

KUMAR, K. K.; RAO, T. V. R. Crop growing periods and irrigation needs of corn crop at some stations in Northeast Brazil. Idojaras, Budapest, v. 111, n. 1, p. 65-77, 2007.

MACHADO, M. A. M. et al. Duração da estação chuvosa em função das datas de início do período chuvoso para o Estado de Minas Gerais. Revista Brasileira de Agrometeorologia, Santa Maria, v. 4, n. 2, p. 73-79, 1996.

MORAIS, A. R. et al. Estimativa da precipitação provável em Lavras (MG) através da distribuição Gama. Revista Brasileira de Agrometeorologia, Santa Maria, v. 9, n. 2, p. 305-310, 2001.
MURTA, R. M. et al. Precipitação pluvial mensal em níveis de probabilidade pela distribuição Gama para duas localidades do sudoeste da Bahia. Ciência e Agrotecnologia, Lavras, v. 29, n. 5, p. 988-994, 2005.

SAMPAIO, S. C. et al. Estimativa e distribuição de precipitações decendiais para o Estado do Paraná. Irriga, Botucatu, v. 12, n. 1, p. 38-53, 2007.

SILVA, J. C. et al. Análise de distribuição de chuva para Santa Maria, RS. Revista Brasileira de Engenharia Agrícola e Ambiental, Campina Grande, v. 11, n. 1, p. 6772, 2007.

SMITH, M. The application of climatic data for planning and management of sustainable rainfed and irrigated crop production. Agricultural and Forest Meteorology, Amsterdan, v. 103, n. 1-2, p. 99-108, 2000.

SOUSA, S. A. V. Programa computacional para simulação da ocorrência de veranicos e queda de rendimento. Pesquisa Agropecuária Brasileira, Brasília, DF, v. 33 n. 12, p. 1952-1956, 1998.

STEWART, J. I. Effective rainfall analysis to guide farm and predict yields. In: ANNUAL GENERAL MEETING OF THE SOIL SCIENCE SOCIETY OF EAST AFRICA, 4., 1980, Arusha. Proceedings... Arusha: SSSEA, 1980. p. 247.

THOM, H. C. S. A note on the Gamma distribution. Monthly Weather Review, Washington, v. 86, n. 4, p. $117-$ $122,1958$. 